\title{
Transfer römischer Technik jenseits der Grenzen: Aneignung und Export
}

\author{
Günther Schörner
}

$1 \quad$ Einführung

Wird das Thema 'Rom jenseits der Grenze' aus archäologischer Perspektive behandelt, so bedeutet dies normalerweise, sich mit so genannten römischen Importen in das Barbaricum zu befassen, also Objekten, die in Italien oder den Provinzen des Römischen Reiches hergestellt wurden und die aus unterschiedlichen Gründen in Gebiete außerhalb des Imperium gelangten. Geläufige Objekte dieser Art sind hochqualitative Keramik, Gefäße oder andere Gegenstände aus Metall, Glas und Münzen¹. Die wissenschaftliche Beschäftigung beschränkt sich nicht mehr nur auf die corpus-mäßige Erfassung

Der vorliegende Aufsatz stellt die erweiterte deutsche Fassung meines Vortrags dar, der unter dem Titel Technology Transfer beyond the Frontiers: Imperial Politics and Local Accomodation am 15. Juni 2013 in New York gehalten wurde. Mein Dank gilt Olivier Hekster (Nijmegen), Daniëlle Slootjes (Nijmegen) und Michael Peachin (New York) für die Einladung zum Elften Workshop des Internationalen Netzwerks ,Impact of Empire. Für wichtige Auskünfte und Kommentare danke ich R. Breitwieser (Salzburg), W. Eck (Köln), M. Speidel (Zürich), K. Sporn (Salzburg/Athen), R. Talbert (Chapel Hill, North Carolina) und N. Wicker (Oxford, Mississippi) sowie meiner Frau H. Schörner.

1 Grundlegende Werke zum so genannten römischen Import: Hans Jürgen Eggers, Der römische Import im Freien Germanien, (Atlas der Urgeschichte) 1 (Hamburg, 1951); Jürgen Kunow, Der römische Import in der Germania libera bis zu den Markomannenkriegen. Studien zu Bronze- und Glasgefäßen, (Göttinger Schriften zur Vor- und Frühgeschichte) 21 (Neumünster, 1983); Ulla Lund Hansen, Römischer Import im Norden, (Nordiske Fortidsminder ser. B) 10 (Kopenhagen, 1987); Lotte Hedeager, „Empire, Frontier and the Barbarian Hinterland: Rome and Northern Europe from $\mathrm{AD} 1-400, "$ in Centre and Periphery in the Ancient World, hrsgg. Michael J. Rowlands, Mogens Larsen Kristian Kristiansen (Cambridge, 1987) S. 125-140; Reinhard Stupperich, „Bemerkungen zum römischen Import im sogenannten Freien Germanien," in Aspekte römisch-germanischer Beziehungen in der frühen Kaiserzeit, hrsg. Georgia Franzius, (Bramsche, 1995) S. 45-98; Reallexikon der Germanischen Altertumskunde Band 25 hrsgg. Heinrich Beck, Dieter Geuenich, Heiko Steuer (Berlin, 2003) S.v. „Römischer Import § 1: Historisches,“ S. 138-143 (Reinhard Wolters); „Römischer Import $\S 2$ : Archäologisches," S. 143-158 (Michael Erdrich - Hans-Ulrich Voß). 
dieser Importe in das Gebiet östlich des Rheins oder nördlich der Donau², sondern bezieht auch andere Regionen und weitergehende Fragestellungen mit ein ${ }^{3}$.

Versteht man den Begriff ,Artefakt ' jedoch in einem weiteren Sinn, so muss er sich nicht auf isolierte Objekte beschränken, sondern kann auch Techniken und die mit ihnen verbundenen Gegenstände - seien es nun einfache Geräte oder komplexere Einrichtungen - umfassen ${ }^{4}$. Die Erweiterung des ArtefaktBegriffs in diese Richtung erlaubt es, Kulturkontakte zwischen Imperium Romanum und indigenen Gesellschaften jenseits der Grenzen auf einer anderen materiellen Basis und mit anderen Fragestellungen zu untersuchen.

Im Folgenden sollen zunächst einige Beispiele für Transfer von Technologie ganz unterschiedlicher Art, mit unterschiedlichem Erfolg und an unterschiedlichen Orten vorgestellt werden ${ }^{5}$; in einem zweiten Teil werden übergeordnete Fragen angesprochen, wobei Anregungen aus dem Bereich der sTs (Science and Technology Studies) aufgegriffen werden ${ }^{6}$.

2 Maßgeblich die Publikationsreihe der Römisch-Germanischen Kommission des Deutschen Archäologischen Instituts, beispielsweise Rudolf Laser, Hans-Ulrich Voß, Corpus der römischen Funde im europäischen Barbaricum D 1: Bundesländer Brandenburg und Berlin (Bonn, 1994); Stefan Berke, Corpus der römischen Funde im europäischen Barbaricum D 7: Land Nordrhein-Westfalen, Landesteile Westfalen und Lippe (Bonn, 2009).

3 Vgl. Reinhard Wolters, „Der Waren- und Dienstleistungsaustausch zwischen dem Römischen Reich und dem Freien Germanien in der Zeit des Prinzipats - eine Bestandaufnahme Teil 1," Münstersche Beiträge zur Antiken Handelsgeschichte 9/1 (1990), 14-44; ders., „Der Waren- und Dienstleistungsaustausch zwischen dem Römischen Reich und dem Freien Germanien in der Zeit des Prinzipats - eine Bestandaufnahme Teil 2," Münstersche Beiträge zur Antiken Handelsgeschichte 10/1 (1991), 78-131. Entscheidend jetzt die Aufsätze in Peter S. Wells, Hrsg., Rome beyond its Frontiers: Imports, Attitudes, and Practices, Supplement Journal of Roman Archaeology 94 (Portsmouth, Rhode Island, 2013) (im Folgenden zitiert als Wells, Rome beyond its Frontiers).

4 Vgl. für diesen Ansatz: Ron Eglash, "Technology as Material Culture," in Handbook of Material Culture, hrgg. Christopher Tilley u.a., (London - Thousand Oaks - New Delhi, 2006) 329-340.

5 Der Begriff, Technologie‘ wird hier in Analogie zum Englischen auch im Deutschen synonym $\mathrm{zu}$,Technik' verwendet und nicht im Sinne einer ,Herstellungs- und Verarbeitungskunde‘.

6 Einführende Literatur:Wenda Bauchspies, Jennifer Croissant, Sal Restivo, Science, Technology, and Society: A Sociological Approach (Malden, Massachusetts - Oxford, 2005); Kevin Greene, "Historiography and Theoretical Approaches," in Handbook of Material Culture, hrsg. John P. Oleson (Oxford, 2008) S. 62-90; Stefan Beck, Jörg Niewöhner, Estrid Sörensen, Science and Technology Studies: Eine sozialanthropologische Einführung (Bielefeld, 2012). Besonders wichtig für die Archäologie ist innerhalb sTs meines Erachtens die Richtung, die auf die soziale Bedingtheit von Techniken hinweist, nämlich Social Construction of Technology (scot) und daraus entwickelte neuere Ansätze; hierfür grundlegend: Wiebe Bijker, Thomas Hughes, 


\section{$2 \quad$ Fallstudien}

\subsection{Pannonisches Limesvorland}

In Cifér-Pác in der heutigen Slowakei wurden in der zweiten Hälfte des 20 Jahrhunderts in einer größeren germanisch geprägten Siedlung eine Gruppe von Häusern freigelegt, deren wichtigstes eine rechteckige Struktur mit Mauern aus Steinen in Mörtelbindung ist, die auf römische Mauertechnik verweist ${ }^{7}$. Im Gebäude wurden zudem ein Ypsilon-förmiger Heizkanal und Fragmente von Tubuli-Ziegeln aufgedeckt, die eine Hypokaustenheizung belegen ${ }^{8}$. Der Kontrast zwischen diesem repräsentativen und gut ausgestatteten Steingebäude und den einfachen Flechtwerkhütten im unmittelbaren Umfeld muss beträchtlich gewesen sein. Einige der Grubenhäuser in germanischer Tradition zeigen freilich eine Kombination unterschiedlicher Baumaterialien,

Trevor Pinch, Hrsgg., The Social Construction of Technological Systems: New Directions in the Sociology and History of Technology (Cambridge, Massachusetts - London, 1987); Pierre Lemonnier, Hrsg., Technological Choices: Transformation in Material Cultures Since the Neolithic: Transformations in Material Cultures Since the Neolithic (London - New York, 1993); Robin Williams - David Edge, "The Social Shaping of Technology," Research Policy 25 (1996), 865-899; Donald MacKenzie, Judy Wajcman, Hrsgg., The Social Shaping of Technology: How the Refrigerator Got Its Hum (Milton Keynes, 1999); Hans K. Klein, Daniel Lee Kleinman, "The Social Construction of Technology: Structural Considerations," Science, Technology \& Human Values 27 (2002), 28-52; Wiebe E. Bijker, "Social Construction of Technology," in: A Companion to the Philosophy of Technology, hrsgg. Jan Kyrre Berg Olsen u.a. (Malden, Massachusetts Oxford, 2009) S. 88-94; Estrid Sörensen, „Die soziale Konstruktion von Technologie (sсOT),“ in Science and Technology Studies: Eine sozialanthropologische Einführung (Bielefeld, 2012) S. 123-144.

7 Zum Fundplatz: Titus Kolník, "Neskororímska vojenská stanica v Páci," Archeologické rozhledy 24 (1972), 59-72. 111-116; ders., "Römische Stationen im slowakischen Abschnitt des nordpannonischen Limesvorlandes," Archeologické rozhledy 38 (1986), 415-420; Lynn F. Pitts, "Roman Style Buildings in Barbaricum (Moravia and sw Slovakia)," Oxford Journal of Archaeology 6 (1987), 234-236; Titus Kolník, "Nordpannonische Limesland-Forschung 1984-1996," in Proceedings of the XVII. International Congress of Roman Frontier Studies, hrsg. Nicolae Gudea (Zalău, 1999) S. 131-137; Titus Kolnik, Jan Rajtár, „Neue Angaben zur Ausdehnung und Funktion der Römisch-germanischen Anlage Cífer-Pác,“ Študijné zvesti archeologického ústavu sav 36 (2004), S. 203-210; Vladimimír Varsik, Titus Kolník, „Cifér-Pác Neue Erkenntnisse zur spätantiken quadischen Elitenresidenz," in Macht des Goldes, Gold der Macht : Herrschafts- und Jenseitsrepräsentation zwischen Antike und Frühmittelalter im mittleren Donauraum Akten des 23. Internationalen Symposiums der Grundprobleme der Frühgeschichtlichen Entwicklung im Mittleren Donauraum, Tengelic, 16. - 19.11.2011, hrsg. Matthias Hardt (Weinstadt, 2013) S. 71-89 (mit der älteren Lit.).

8 Kolnik, Rajtár, „Neue Angaben,“ S. 203. 
da sie mit tegulae gedeckt waren, so dass römische Bautechnik nicht auf das bereits erwähnte Gebäude beschränkt bleibt.

Die Siedlung kann durch Keramik und Münzen in die Zeit vom frühen 2. bis zum späteren 4. Jahrhundert n. Chr. datiert werden, wobei die meisten der früheren Funde aus den mit herkömmlichen Materialien konstruierten Hütten stammen, während für die aufwändigen Strukturen eine Errichtung in der Spätzeit der Siedlung wahrscheinlich ist ${ }^{9}$. Daraus lässt sich schließen, dass in einer seit dem 2. Jahrhundert n. Chr. existierenden germanischen Siedlung in Cífer-Pác in der 1. Hälfte des 4. Jahrhunderts eine massive Umgestaltung erfolgte, indem eine große Anlage in römischer Steinbauweise und mit einer Heizung römischen Typs errichtet wurde und für einfachere Häuser partiell römische Bautechnik angewandt wurde, die mit Dachziegeln anstelle Stroh gedeckt waren. Die Errichtung dieser Gebäude und damit die Implementierung römischer Bautechnik fallen in eine Zeit, als römische Militärkampagnen jenseits der Donau durchgeführt wurden, wie Ammianus Marcellinus schreibt ${ }^{10}$. Von besonderer Wichtigkeit für die Bewertung des Technologietransfers ist die funktionale Charakterisierung Cifér-Pács: Die Tradition als indigener Siedlungsplatz und das Fehlen spezifisch fortifikatorischer Bauten sprechen gegen eine Interpretation als römischer Militärposten, sondern eher für einen besonders herausgehobenen Wohnsitz eines lokalen Potentaten, der mit Hilfe römischer Truppen errichtet wurde, wobei auch für einfachere Architekturen partiell römische Baumaterialien adaptiert wurden ${ }^{11}$.

Eine ähnliche Konstellation können wir an einem anderen Ort im Vorfeld des pannonischen Limes greifen, nämlich Bratislava-Dúbravka in der Nähe des Zusammenflusses von Donau und March. Wie Cifér-Pác bestand der Siedlungsplatz in einer ersten Phase seit der 2. Hälfte des 1. Jahrhunderts n. Chr. aus einer kleinen Ansammlung von Grubenhäusern mit Wänden aus Flechtwerk und strohgedeckten Dächern ${ }^{12}$. Dieser relativ einfache, indigene

9 Ibid.

$10 \quad$ Ammianus Marcellinus XXIX 6.

11 Symptomatisch die Neubewertung des Fundplatzes in den Schriften von Titus Kolník. Kolník, „Stanica“: römische Militärstation; Kolník, „Römische Stationen“: polyfunktionelle Anlage; Kolník, „Limesland-Forschung“: germanische Residenz.

12 Zum Fundplatz: Kolník, „Römische Stationen,“ 431; Janka Hečková, „Römischer Baukomplex in Stupava“, Archeologické rozhledy 38 (1986), 392; Pitts, „Roman Style Buildings“, 227-229; Kristian Elschek, „Die germanische Besiedlung von BratislavaDúbravka während der älteren römischen Kaiserzeit," in Kelten, Germanen, Römer im Mitteldonaugebiet, hrsgg. Jaroslav Tejral u. a. (Brünn - Nitra, 1995) S. 39-52; Kristian Elschek, „Römisch-germanische villae rusticae im Limesvorfeld von Carnuntum? Ergebnisse systematischer Grabung und Prospektion," in: Roman Frontier Studies 1995 
Charakter wurde während der nächsten Phase, die aufgrund von Keramikund Münzfunden in die 2. Hälfte des 3. Jahrhunderts datiert werden kann, beträchtlichen Veränderungen unterworfen, unter denen die Errichtung eines $13 \times 11 \mathrm{~m}$ großen Gebäudes in Steinbautechnik die wichtigste war ${ }^{13}$. Die Ziegel zur Eindeckung dieses im Siedlungskontext sehr eindrucksvollen Gebäudes wurden aus der römischen Provinz Pannonien importiert, wie Ziegelstempel belegen ${ }^{14}$. Besonders auffällig ist das Gebäude aufgrund seines für die Region außergewöhnlichen Grundrisses mit drei Apsiden, für den sich aber in den römischen Nordprovinzen enge Parallelen finden lassen, da bei Thermenanlagen von Villen Frigidarium, Tepidarium und Caldarium häufig absidal abgeschlossen sind ${ }^{15}$. Trotz dieser formalen Übereinstimmung zwischen dem Gebäude in Bratislava-Dúbravka und den provinzialen Badeanlagen gibt es einen fundamentalen Unterschied: Es existiert nicht der geringste Hinweis darauf, dass das Gebäude im Limesvorland beheizt war, wie es für eine Nutzung als Therme unbedingt zu fordern ist ${ }^{16}$. Es kann nur spekuliert werden, weshalb keine Heizung eingebaut wurde, ob beispielsweise der ursprüngliche Bauplan aufgegeben wurde. Zweifelsfrei steht jedoch fest, dass es anders genutzt werden musste als seine formalen Vorbilder. Obwohl das Gebäude wie ein römisches Bad ausgesehen hat, wie sie in den Nordprovinzen von zahlreichen Exemplaren bekannt sind, konnte es nicht dieselben Funktionen eines temperaturmäßig ausdifferenzierten Bades erfüllen, so dass das Gebäude in Bratislava-Dúbravka zugespitzt als Thermenattrappe bezeichnet werden könnte.

(Oxford, 1997) S. 225-232; Kristian Elschek, „Ausgewählte römische Fibeltypen vom slowakischen Marchgebiet im Limesvorland von Carnuntum und ein neues Fürstengrab der "Lübsow-Gruppe" von Zohor," in Kelten, Germanen, Römer im Mitteldonaugebiet, hrsgg. Gerald Grabherr, Barbara Kainrath, Thomas Schierl (Innsbruck 2013) S. 195 (mit der älteren Lit.).

13 Zur Datierung: Elschek, „Römisch-germanische villae rusticae,“ 225.

14 Elschek, „Römisch-germanische villae rusticae,“ 228. So ist Septimius Vitalis auch aus Vindobona bekannt: Friedrich Kenner, Bericht ueber roemische Funde in Wien in den Jahren 1896-1900 (Wien, 1900) S. 7 Abb. 4; 24.

15 Zu Thermen in den benachbarten Provinzen Noricum und Pannonien: Stefan Traxler Raimund Kastler, Hrsgg., Römische Bäder in Raetien, Noricum und Pannonien. Colloquium Lentia 2010. Beiträge zur Tagung im Schlossmuseum Linz 6.-8. Mai 2010 (Studien zur Kulturgeschichte von Oberösterreich) 27 (Linz, 2012).

16 Kristian Elschek, „Rímsko-germánska vidiecka usadlost' s kúpel’om v Bratislave-Dúbravke,“ Pamiatky a múzeá 3 (2000), 27-29; vorsichtiger: Pitts, „Roman Style Buildings“, 228 (ungenutztes praefurnium, Bau evtl. unfertig). 


\subsubsection{Keramikherstellung}

Ein besonders vielschichtiges Beispiel für Technologietransfer stellt die Töpferwerkstatt im thüringischen Haarhausen dar. In diesem Ort im heutigen Thüringen wurden von Sigrid Dušek in den 1980er Jahren drei Töpferöfen und weitere Anlagen zur Keramikproduktion ausgegraben ${ }^{17}$. Alle Öfen sind frei stehende Anlagen und setzen sich aus einem Feuerungskanal, einem Feuerungsraum und einem Brennraum zusammen, die durch eine Lochtenne voneinander getrennt sind ${ }^{18}$. Die Gestalt der Öfen zeigt einen charakteristischen Grundriss in Form eines Schlüssellochs, da der Feuerungskanal schmaler ist als der Brennraum und dieser durch eine Wand noch partiell zweigeteilt ist. Während diese unteren Partien aus mit Stroh gemagertem Lehm bestehen, wurde die Kuppel des Brennraums aus ineinander gefügte Gefäße, die mit Lehm verstrichen sind, errichtet und abschließend mit Kalkmörtel verputzt. Die durch diese konstruktiven Details charakterisierte Bauweise der Töpferöfen hat keine Parallelen außerhalb des Imperium Romanum, da weder im näheren wesergermanischen und elbgermanischen Bereich noch in den weiter entfernten großen Töpfereizentren in Polen und Mähren vergleichbare Konstruktionen gefunden wurden ${ }^{19}$. Innerhalb des Imperium Romanum gibt

17 Sigrid Dušek, „Die Produktion römischer Drehscheibenkeramik in Thüringen Technologie, ökonomische und gesellschaftliche Konsequenzen," in Römerzeitliche Drehscheibenkeramik im Barbarikum. Symposium Weimar, 21. bis 25. Mai 1984., hrsg. Rudolf Feustel, Weimarer Monografien zur Ur- und Frühgeschichte 11 (Weimar, 1984) S. 6-18; dies., „Produktion und Verbreitung römischer Reibschalen im hermundurischen Thüringen,“ Acta Rei Cretariae Romanae Fautores 29/30 (1991), 137-148; dies., Römische Handwerker im germanischen Thüringen. Ergebnisse der Ausgrabungen in Haarhausen, Kreis Arnstadt. Teil A: Auswertung (Stuttgart, 1992) (im Folgenden zitiert als Dušek, Haarhausen); dies., Römische Handwerker im germanischen Thüringen. Ergebnisse der Ausgrabungen in Haarhausen, Kreis Arnstadt. Teil B: Fundbericht (Stuttgart, 1992); Oliver Stoll, „Der Transfer von Technologie in der römischen Antike: einige zusätzliche Bemerkungen zu einem Buch von Sigrid Dušek," Münstersche Beiträge zur Antiken Handelsgeschichte 12/2 (1993), 93-118; Sigrid Dušek, Ur- und Frühgeschichte Thüringens (Stuttgart, 1999) S. 134137; Bernd Steidl, „Lokale Drehscheibenkeramik römischer Formgebung aus dem germanischen Mainfranken. Zeugnis für die Verschleppung römischer Reichsbewohner nach Germanien?," Bayerische Vorgeschichtsblätter 67 (2002), 87f. und passim (im Folgenden zitiert als Steidl, „Drehscheibenkeramik“).

18 Dušek, Haarhausen, S. 12-17,

19 Dušek, Haarhausen, S. 52-66. Zur eventuellen Tätigkeit provinzialrömischer Töpfer in Südpolen: Pavel Wielkowiejski, "Przemiany gosdpodarczo-spoleczne u ludności południowej Polski w okresie późno-latenskim i rzymskim," Materiaty Starožytne 6 (1960), 119); in der Ostslowakei Pavel Jurečko, „Problematika tzv. siwej keramiky v dobe rimskej 
es jedoch zahlreiche Parallelen für die Haarhausener Öfen. So wurden in NidaHeddernheim in der Provinz Germania superior Anlagen aufgedeckt, die in Gestaltung, Konstruktionstechnik und verwendeten Materialien, insbesondere den Wölbtöpfen, sehr gut vergleichbar sind ${ }^{20}$. Die Ähnlichkeiten sind so stark, dass S. Dušek Haarhausen als einen Filialbetrieb der Heddernheimer Töpfer bezeichnet; diese Fokussierung auf einen spezifischen Herkunftsort wird jedoch in jüngerer Zeit abgelehnt ${ }^{21}$. Eindeutig ist jedoch der weitreichende Transfer römischer Technik für die Konstruktion von Töpferöfen, wobei sich die Implementierung römischen Knowhows nicht auf diesen Bereich beschränkt. Auch die Errichtung eines $12 \times 5 \mathrm{~m}$ großen Gebäudes, das wohl als Trocken- und Arbeitshalle diente, verweist auf die Übernahme provinzialer Bautechnik ${ }^{22}$. Der Ausstoß der Töpferei in Haarhausen wurde aufgrund der Anzahl der Öfen, ihrer Größe und der vermutlichen Anzahl von möglichen Brennvorgängen auf ca. 60.000-70.00o Gefäßen pro Jahr geschätzt, woran 25 bis 30 Töpfer und weitere Arbeitskräfte beteiligt gewesen sein könnten ${ }^{23}$.

Beim Brennen kam ebenfalls römische Technologie zur Anwendung, da ringförmige Abstandhalter und auch Ständer gefunden wurden, die Parallelen in Nida-Heddernheim besitzen ${ }^{24}$. Der Brandvorgang selbst fand in einer sauerstoffarmen, also reduzierenden Atmosphäre statt und unterschied sich dadurch zur gängigen Praxis im Imperium Romanum ${ }^{25}$. Dies bedeutet, dass die Gefäße einen grauen bis schwarzen Scherben bei einer lederfarbigen bis schwarzen Oberfläche aufweisen im Kontrast zu den beige-rötlichen Erzeugnissen der römischen Werkstätten.

In Haarhausen wurde überwiegend Keramik auf der schnell drehenden Töpferscheibe hergestellt, nur ca. ein Viertel der gefundenen Scherben gehört zu frei geformten Gefäßen, die also in der im rhein-wesergermanischen Kreis üblichen Produktionstechnik gefertigt wurden. Die Gefäße entsprechen zum einen bekannten germanischen Typen, ersetzen also ohne Töpferscheibe hergestelltes Geschirr durch qualitativ höherwertige Drehscheibenkeramik, zum anderen folgen sie formal Produkten aus dem Imperium Romanum, so

so zretelom na výsledky výskumu na východnom Slovensku," Historica Carpatica 12 (1981), 169-209.

20 Dušek, Haarhausen, S. 56f.; zu Töpferöfen in Nida-Heddernheim: Susanne Biegert, Römische Töpfereien in der Wetterau (Frankfurt; 1999).

21 Steidl, „Drehscheibenkeramik," S. 107 Anm. 47.

22 Dušek, Haarhausen, S. 17-19.

23 Dušek, Haarhausen, S. 98-101.

24 Dušek, Haarhausen, S. 68f.

25 Signifikant ist die germanische Terra nigra-Keramik: Dušek, Haarhausen, S. 93-98; vgl. jedoch die kritischen Bemerkungen bei Steidl, „Drehscheibenkeramik,“ S. 99f. Anm. 25. 
dass insbesondere Krüge und Reibschalen (mortaria) zum Repertoire der Handwerker in Haarhausen gehören ${ }^{26}$. Für die römischen Gefäßformen lässt sich kein einheitlicher Ursprungsort festmachen, da anscheinend Vorlagen aus verschiedenen Regionen verwendet wurden und Parallelen in Germania inferior, Germania superior, Gallia belgica und Raetia nachzuweisen sind.

Die Produktionsstätte in Haarhausen kann durch Kleinfunde, vor allem Fibeln des Typs Almgren 175, 177 und 181 sowie 14 C-Datierungen in die Jahre zwischen 260 und 290 gesetzt werden ${ }^{27}$. Die Töpferei war also nur in einer Zeit in Betrieb, als der Obergermanisch-rätische Limes bereits gefallen war und als das Imperium Galliarum zumindest zeitweise der bestimmende Faktor in der Region war' ${ }^{28}$.

Keramik aus Haarhausen wurde in mehr als 150 Orten überwiegend in Thüringen gefunden, wurde aber wahrscheinlich bis nach Mainfranken verhandelt ${ }^{29}$. Gefäße der Haarhausener Produktion sind auch in den Fürstengräbern von Hassleben-Leuna bezeugt, die in die Zeit von 260 bis $310 \mathrm{n}$. Chr. datiert werden können, so dass der vor Ort gewonnene Ansatz bestätigt wird ${ }^{30}$.

Zweifellos liegt in Haarhausen eine besonders komplexe Form von Technologietransfer vor, da folgende römische Techniken bezeugt sind:

1. Konstruktion von Töpferöfen

2. Produktion von Drehscheibenkeramik

3. Beschickung der Töpferöfen und auch teilweise der Brennvorgang

Die Töpferwerkstatt in Haarhausen war ein relativ kurzzeitiges Phänomen. Die Produktion fällt in die 2. Hälfte des 3. Jahrhunderts n. Chr., so dass Gefäße in römischer Formtradition nur eine Generation lang bis zu Beginn des 4 . Jahrhunderts hergestellt werden. Die Verwendung der Töpferscheibe setzt sich

26 Dušek, Haarhausen, S. 70-93; vgl. aber auch die Bemerkungen bei Steidl, „Drehscheibenkeramik," S. 103-105.

27 Dušek, Haarhausen, S. 51 f.

28 Zum Imperium Galliarium: John F. Drinkwater, The Gallic Empire. Separatism and Continuity in the North-Western Provinces of the Roman Empire A.D. 260-274, (Historia Einzelschriften) 52 (Stuttgart, 1987); Thomas Fischer, Hrsg., Die Krise des 3.Jahrhunderts $n$. Chr. und das Gallische Sonderreich (Wiesbaden, 2012) (mit der weiteren Lit.).

29 Dušek, Haarhausen, S. 103-115; Beleg in Mainfranken: Steidl, „Drehscheibenkeramik,“ S. 100.105 .

$30 \quad$ z.B.W. Schulz, Das Fürstengrab von Hassleben, (Römisch-Germanische Forschungen) 7 (Berlin - Leipzig, 1933) S. 6. 21. 51; vgl. auch Steidl, „Drehscheibenkeramik,“ S. 110 mit Anm. 57 . 
jedoch über das 4. Jahrhundert bis in das 5. Jahrhundert hinein fort, wobei dann aber nur noch germanische Gefäßtypen wie Flaschen oder so genannte Schalenurnen fabriziert wurden ${ }^{31}$.

Es ist der Ausgräberin zuzustimmen, dass aufgrund dieser Massierung römischen Know-hows davon ausgegangen werden muss, dass Handwerker aus dem Imperium Romanum in Haarhausen gearbeitet haben. Ob ihr jedoch auch mit der weiteren Schlussfolgerung recht zu geben ist, nämlich dass die Töpfer die Provinz verließen, um ein sicheres Arbeitsumfeld zu finden und neue Absatzmärkte zu erschließen, ist fraglich ${ }^{32}$. Ausgangspunkt für diese Überlegung ist eine Stelle bei Tacitus, die besagt, dass die Hermunduren in einer besonderen Beziehung zum Imperium standen und auch Vorrechte beim Handel mit dem Römischen Reich genossen ${ }^{33}$. Abgesehen davon, dass die von Tacitus geschilderten Verhältnisse nicht mit denen des fortgeschrittenen 3. Jahrhunderts übereinstimmen müssen und ganz zu schweigen von den Schwierigkeiten, mit ,Stämmen' als festen Gruppen zu operieren, ist es nicht wahrscheinlich, dass die, Römer' ganz ohne fremde Hilfe gearbeitet haben können, da Dušeks Ansatz die Notwendigkeit unterschlägt, dass die Infrastruktur Arbeitskräfte, Rohmaterialien usw. - für ortsunkundige Neuankömmlinge von den Einheimischen zur Verfügung gestellt werden mussten ${ }^{34}$. Aber auch die andere bisher geäußerte These, dass es sich um kriegsgefangene Provinziale gehandelt habe, die nach Germanien verschleppt wurden, aber trotzdem die Gefäße römischen Typs für den eigenen Bedarf hergestellt hätten, ist nicht restlos überzeugend ${ }^{35}$. Vor allem ist gegen diese Hypothese anzuführen, dass die zwangsverschleppten Töpfer doch über einen nicht wirklich begründbaren großen Handlungsspielraum verfügt haben müssten. Beide Annahmen sehen die Handlungsmacht vor allem bei den römischen Handwerkern, während die indigenen Akteure zu wenig berücksichtigt werden. Die entscheidende Kraft

31 Steidl, „Drehscheibenkeramik,“ S. 105f.

32 Dušek, Haarhausen, S. 132-148.

33 Tac. Ger. 41.

34 Eine ethnologische Deutung römischer Schriftquellen insbesondere über einen mehrere Jahrhunderte dauernden Zeitraum wird mittlerweile von der Forschung abgelehnt. Zum problematischen ,ethnologischen' Konzept der ,Stämme' wie der Hermunduren: Tom Moore, "Detribalizing the Later Prehistoric Past: Concepts of Tribes in Iron Age and Roman Studies," Journal of Social Archaeology 11 (2011), 334-360.

35 So jedoch B. Schmidt, „Drehscheibenkeramik der spätrömischen Kaiserzeit und frühen Völkerwanderungszeit im Mittelelb-Saalegebiet," in Römerzeitliche Drehscheibenkeramik im Barbarikum. Symposium Weimar, 21. bis 25. Mai 1984, hrsg. Rudolf Feustel, Weimarer Monografien zur Ur- und Frühgeschichte 11 (Weimar, 1984) S. 26; Steidl, „Drehscheibenkeramik," S. 108-111. 
ist wohl eher in den einheimischen Eliten zu sehen, die für die Ansiedlung fremder Produzenten verantwortlich gemacht werden können, da sie allein über die Mittel verfügten, eine solch umfangreiche Produktion zu etablieren und mit der nötigen Infrastruktur auszustatten.

Eine wichtige Ergänzung erfuhren die Befunde in Haarhausen durch die Forschungen Bernd Steidls zu römischen Funden in Mainfranken, durch die eine von Haarhausen unabhängige, aber gleichzeitige Produktion von Drehscheibenkeramik nachgewiesen wurde $^{36}$. Der Töpferbetrieb konnte zwar bisher nicht identifiziert werden, doch sprechen Altfunde für eine Lokalisierung in Eßleben bei Schweinfurt ${ }^{37}$. Weil jedoch die Töpferöfen und auch die sonstige Infrastruktur samt Brennhilfen unbekannt sind, kann nicht davon ausgegangen werden, dass sie wie in Haarhausen römischer Technologie folgten. Sicher ist jedoch, dass Drehscheibenkeramik in römischer Machart hergestellt wurde. Detaillierte Untersuchungen an der stark fragmentierten Keramik ergaben signifikante Modifikationen bei der Produktion ${ }^{38}$ :

1.) Die Magerung der Gefäße ist nicht einheitlich. Sie kann teilweise sehr fein sein und somit dem römischen Usus entsprechen, aber auch aus gröberen Material bestehen und daher eher der germanischen Praxis folgen

2.) Die Behandlung der Oberfläche variiert stark. Neben unbehandelten Oberflächen kommt auch Glättung mit einem Polierstein vor, wie sie für germanische Terra nigra-Keramik üblich ist.

3.) Der Brand erfolgte in reduzierender Atmosphäre, so dass sich die Gefäße - wie auch die Haarhausener Stücke - in der Farbe deutlich von oxidierend gebrannter, also orange-roter Keramik unterscheiden, wie sie für provinzialrömische Produktion charakteristisch ist.

4.) Allgemein orientierte man sich bei der Formenwahl eher an römischen Traditionen. Exakte Parallelen für die einzelnen Gefäßtypen in den benachbarten römischen Provinzen ließen sich jedoch nicht feststellen.

5.) Typisch römische Gefäßformen werden signifikant verändert, wie man besonders gut an drei mortaria aus Eßleben und Frankenwinheim sehen $\mathrm{kann}^{39}$. Sie weisen einen Standring auf, der bei originalen römischen Reibschalen selten vorkommt. Zudem wurde die Arbeitsfläche im Inneren signifikant umgestaltet: Die Reibfläche im Inneren wurde

36 Steidl, „Drehscheibenkeramik,“ S. 87-116.

37 Steidl, „Drehscheibenkeramik,“ S. 105.

38 Steidl, „Drehscheibenkeramik," S. 98-100.

39 Steidl, „Drehscheibenkeramik,“ S. 92 Nr. 11 Abb. 3, 11; 94 Nr. 36-37 Abb. 4, 36-37; 99 Taf. 8, $11.36-37$. 
anstelle von grobem mit feinem Sand bestreut, zudem tief in die Wandung eingedrückt und teilweise mit Ton überschmiert. Dies bedeutet, dass die Gefäße nicht in ihrer sonst üblichen Funktion als Reibschalen verwendet werden konnten.

Diese Modifikationen zeigen, dass sich die Gefäße von römischen Vorbildern unterschieden und dass folglich auch die Herstellungsprozesse differierten. Steidl nimmt auch für die mainfränkischen Betriebe an, dass hier römische Gefangene als Töpfer tätig waren ${ }^{40}$. Dies ist möglich, doch sind die geschilderten Anpassungen nicht denkbar, ohne dass eine enge Interaktion mit indigenen Handwerkern auch im Sinne einer Zusammenarbeit stattgefunden hat. Vielleicht ist doch an Technologietransfer durch römisch ausgebildete germanische Töpfer zu denken, zumal anders als in Haarhausen der Beweis dafür fehlt, dass auch komplizierte Produktionseinrichtungen wie Töpferöfen exakt nach römischen Vorlagen errichtet wurden.

\subsubsection{Bleigewinnung}

Eine der für Technologietransfer wichtigsten archäologischen Entdeckungen in den letzten Jahren ist der Nachweis, dass im rechtsrheinischen Germanien sowohl unter römischer Verwaltung als auch nach Abzug römischer Truppen über den Rhein Blei produziert wurde ${ }^{41}$. Bereits kurz nach den augusteischen Feldzügen muss die Ausbeutung von entsprechenden Lagerstätten im Bergischen Land bei Bonn und vor allem im Hochsauerland bei Brilon eingesetzt haben ${ }^{42}$. Da Bleigewinnung in der vorrömischen Zeit nicht nachgewiesen ist und anscheinend abrupt begann, ist davon auszugehen, dass römische Prospektoren den Legionen unmittelbar folgten oder sogar vorausgegangen sind.

Das unter römische Verwaltung gewonnene Blei wurde über Gallien nach Rom bzw. an das Mittelmeer geschafft, wie dies Schiffswracks in Südfrankreich

40 Steidl, „Drehscheibenkeramik,“ S. 108-111.

41 Zum Bleibergbau in Germanien allgemein: Walter Melzer, Torsten Capelle (Hrsg.), Bleibergbau und Bleiverarbeitung während der römischen Kaiserzeit im rechtsrheinischen Barbaricum, (Soester Beiträge zur Archäologie) 8 (Soest, 2007) (im Folgenden zitiert als Melzer, Capelle, Bleibergbau).

42 Zum Bergbau auf dem Lüderich im Bergischen Land: Gabrielle Körlin, Michael Gechter, „Römischer Bergbau auf dem Lüderich. Vorbericht über die Grabungen 2000 bis 2002," in Man and Mining - Mensch und Bergbau. Studies in Honour of G. Weisgerber on Occasion of his 65th Birthday, hrsgg. Thomas Stöllner u. a. (Bochum, 2003) S. 237-248. 
bei Saintes Maries-de-la-mer und Sardinien beweisen ${ }^{43}$. Die Stempel auf den Bleibarren zeigen, dass sowohl der Kaiser selbst als auch private Unternehmer die Vorkommen ausbeuteten ${ }^{44}$. Die Herkunft aus Germanien ist sowohl durch das Kürzel GERM für plumbum germanicum als durch neue naturwissenschaftliche Untersuchungen gesichert ${ }^{45}$. Die Bleibarren haben die übliche römische Form, wie wir sie auch aus Britannien oder Spanien kennen, und folgen auch dem römischen Standardgewicht von 195 römischen Pfund $(=64 \mathrm{~kg})^{46}$.

Durch Lesefunde, aber auch durch Ausgrabungen in germanischen Siedlungen sind aber auch zahlreiche kleinere Bleibarren bekannt ${ }^{47}$, die wesentlich leichter als ihre römischen Gegenstücke sind, nämlich nur 300 bis $600 \mathrm{~g}$, wobei das Gewicht stark variieren $\mathrm{kann}^{48}$. Auch formal unterscheiden

43 Françoise Laubenheimer-Leenhardt, Recherches sur les lingots de cuivre et de plomb d'époque romaine dans les regions de Languedoc-Roussillon et de Provence-Corse, 3, (Supplément Revue Archéologique Narbonnaise) 3 (Paris, 1973) S. 124f. Nr. 16; 195-197; Norbert Hanel, Peter Rothenhöfer, „Germanisches Blei für Rom. Zur Rolle des römischen Bergbaus im rechtsrheinischen Germanien im frühen Prinzipat," Germania 83 (2005), 58-6o; Norbert Hanel, Peter Rothenhöfer, „Römische Bleigewinnung im Raum Brilon und der Bleitransport nach Rom, "in: Melzer, Capelle, Bleibergbau, S. 41-46.

44 Als private Unternehmer sind Flavius Verucla und Pudens bezeugt: Norbert Hanel, Peter Rothenhöfer, „Germanisches Blei für Rom. Zur Rolle des römischen Bergbaus im rechtsrheinischen Germanien im frühen Prinzipat," Germania 83 (2005), 59 .

45 Inschrift: Norbert Hanel, Peter Rothenhöfer, „Römische Bleigewinnung im Raum Brilon und der Bleitransport nach Rom," in: Melzer, Capelle, Bleibergbau, S. 42-44; naturwissenschaftliche Untersuchungen: Michael Bode, Archäometallurgische Untersuchungen zur Blei-/Silbergewinnung im Germanien der frühen Römischen Kaiserzeit (Münster, 2008) S. 134-138 Abb. 5.8. 5.9.

46 Zur Typologie römischer Bleibarren: Claude Domergue, Les mines de la Péninsule ibérique dans l'antiquité romaine (Collection École Française de Rome) 127 (Rom, 1990) S. 253f.; Norbert Hanel, Peter Rothenhöfer, „Germanisches Blei für Rom. Zur Rolle des römischen Bergbaus im rechtsrheinischen Germanien im frühen Prinzipat," Germania 83 (2005), 58 f. $\mathrm{Zu}$ Bleifunden in indigenen Siedlungen der älteren Römischen Kaiserzeit: Peter Rothenhöfer, „Das Blei der Germanen. Bemerkungen zu einer neuen Fundgattung und zur Aufnahme der Bleiproduktion durch Germanen während der älteren Römischen Kaiserzeit in Westfalen," Archäologisches Korrespondenzblatt 34 (2004), 423; Peter Rothenhöfer, „Iam et plumbum excoquere docuimus? - Zum Phänomen der germanischen Bleiproduktion im nördlichen Sauerland während der römischen Kaiserzeit," in Melzer, Capelle, Bleibergbau, S. 47-49 Abb. 2 (Übersicht); Martin Straßburger, „Plumbi nigri origo duplex est - Bleierzbergbau der römischen Kaiserzeit im nordöstlichen Sauerland," in Melzer, Capelle, Bleibergbau, S. 6o-62; Bode, Untersuchungen, S. 112f.

48 Rothenhöfer, „Blei der Germanen,“ S. 423-426 Abb. 2; Peter Rothenhöfer, „Iam et plumbum excoquere docuimus? - Zum Phänomen der germanischen Bleiproduktion im nördlichen Sauerland während der römischen Kaiserzeit," in Melzer, Capelle, Bleibergbau, 
sie sich deutlich von diesen Standardbarren, da sie einen trapezförmigen bis rechteckigen Umriss besitzen und an einer Schmalseite eine Durchbohrung aufweisen. Anders als man lange Zeit glaubte, gehören diese Barren nicht in das Mittelalter, sondern sind aufgrund des Fundkontextes in die römische Kaiserzeit, genauer in das 1. und 2. Jahrhundert n. Chr., vielleicht auch noch in das 3. Jahrhundert n. Chr., zu datieren ${ }^{49}$. Die Zeitstellung und die vollkommen andere Gestaltung der Barren sprechen also für eine nicht-römische, das heißt germanische Bleiproduktion, die aufgrund fehlender eigener Tradition auf römischer Bergbau- und Verhüttungstechnologie fußen muss. Im Einzelnen waren folgende Arbeitsschritte zur Gewinnung von Blei aus Bleiglanz, dem lokal vorkommenden Erz, erforderlich ${ }^{50}$ :

- Prospektion und Abbau des Erzes

- trockenmechanische Aufbereitung

- Schweretrennung im Wasser

- Rösten des Erzes

- Reduktion des Bleioxids zu Blei

- Weiterverarbeitung zu Barren

Der Arbeitsvorgang ist aufwändig, aber nicht besonders schwierig, so dass ein Technologietransfer durch Nachahmung möglich gewesen sein kann, beispielsweise dadurch, dass indigene Arbeitskräfte durch ihre Tätigkeit in römischen Betrieben sich das entsprechende Know-how abgeschaut haben ${ }^{51}$. Freilich ist davon auszugehen, dass die Technik im Einzelnen abgewandelt wurde. Unklar muss bleiben, ob sich die Abbautechniken verändert haben, da sicher mit diesem germanischen Abbau zu verbindende Spuren noch

S. 47-51 Abb. 1. 3. 4. 6; 54f. (Katalog). Vgl. auch Michael Erdrich, „Rezension zu Walter Melzer - Torsten Capelle (Hrsg.), Bleibergbau und Bleiverarbeitung während der römischen Kaiserzeit im rechtsrheinischen Barbaricum," Germania 87 (2009), 629.

49 Dies gilt für die Fundorte Salzkotten-Thüle, Balve-Garbeck, Brilon-Fülsenbecke und vor allem Soest: Bode, Untersuchungen, S. 112 (mit der weiteren Lit.). Zur Datierung: Peter Rothenhöfer, „Iam et plumbum excoquere docuimus? - Zum Phänomen der germanischen Bleiproduktion im nördlichen Sauerland während der römischen Kaiserzeit," in Melzer, Capelle, Bleibergbau, S. 51; Martin Straßburger, „Plumbi nigri origo duplex est Bleierzbergbau der römischen Kaiserzeit im nordöstlichen Sauerland," in Melzer, Capelle, Bleibergbau, S. 6o-62.

5o Zu technisch-chemischen Vorgängen der Bleigewinnung: Bode, Untersuchungen, S. 8-10.

51 Peter Rothenhöfer, „Iam et plumbum excoquere docuimus? - Zum Phänomen der germanischen Bleiproduktion im nördlichen Sauerland während der römischen Kaiserzeit," in Melzer, Capelle, Bleibergbau, S. $5^{2 f}$. 
nicht entdeckt wurden ${ }^{52}$. Die nächsten Produktionsschritte müssen jedenfalls gleich geblieben sein, da sonst nicht das gewünschte Ergebnis - Blei hätte erzielt werden können. Denkbar sind freilich kleinere Modifikationen bei einzelnen Komponenten wie Werkzeugen. Die größte Veränderung, die eindeutig archäologisch nachweisbar ist, betrifft den letzten Arbeitsgang, die Weiterverarbeitung zu Barren, da deren Form sich deutlich von den römischen Barren unterscheidet. Das kleinere Gewicht und die fehlende Standardisierung sprechen für einen geringeren Grad bei der Organisation des germanischen Bleiabbaus, da vielleicht jeder einzelne Betrieb seine eigene Barrengröße wählte, und für eine schlechtere Infrastruktur entweder bei der Bleiproduktion selbst, da nur kleinere Mengen Blei gewonnen wurden oder bei der Verkehrserschließung, weil leichtere Barren natürlich auch einfacher zu transportieren waren. Der letzte Aspekt ist deswegen wichtig, weil das gewonnene Blei mit hoher Wahrscheinlichkeit in das Imperium Romanum verhandelt wurde, obwohl auch sporadisch indigene Buntmetallverarbeitung im rechtsrheinischen Germanien nachgewiesen werden kann ${ }^{53}$.

\subsection{Fezzan}

Als eine besonders ergiebige Region für den Nachweis von Technologietransfer erwies sich der Fezzan, obwohl diese hyperaride Region im südlichen Libyen besonders weit vom Imperium Romanum entfernt war. Der Fezzan (Oase alAjal) gehörte während der gesamten Antike, also von ca. $500 \mathrm{v}$. Chr. bis $500 \mathrm{n}$. Chr., zum Siedlungsgebiet der Garamanten, und hatte sein politische Zentrum in Gerama (Jarma), das ungefähr 10ookm von der Küste des Mittelmeers entfernt liegt, was einer Reise von 30 Tagen mit einer Karawane entspricht ${ }^{54}$. Aufgrund intensiver archäologischer Feldforschungen während der italienischen Okkupation in den 3oer Jahren und libysch-britischer Forschungen seit den 7oer Jahren des letzten Jahrhunderts ist die materielle Kultur der Garamanten gut bekannt ${ }^{55}$.

52 Martin Straßburger, „Plumbi nigri origo duplex est - Bleierzbergbau der römischen Kaiserzeit im nordöstlichen Sauerland," in Melzer, Capelle, Bleibergbau, S. 62 f.

53 Bleiverarbeitung vor allem in Soest bezeugt: hier Anm. 47. Verhandlung in das Imperium: Erdrich, „Rezension,“ S. 629f. Kritisch: Martin Straßburger, „Plumbi nigri origo duplex est Bleierzbergbau der römischen Kaiserzeit im nordöstlichen Sauerland, in Melzer, Capelle, Bleibergbau, S. 65-68.

54 Zum Fezzan allgemein: Nick Brooks u. a., "Studies in Geography, Geomorphology, Environment and Climate," in The Archaeology of Fazzan. Volume 1, Synthesis, hrsg. David J. Mattingly (London - Tripoli, 2003) S. 37-74 (im Folgenden zitiert als Mattingly, Synthesis). Erwin W. Ruprechtsberger, Die Garamanten. Geschichte und Kultur eines libyschen Volkes in der Sahara. Sonderhefte Antike Welt (Mainz 1997), S. 29-50; David J. Mattingly - Tim 
Die Ausgrabungen in Gerama deckten eine Siedlung auf mit durchaus urbanem Charakter, die neben einfacheren Strukturen aus einer zentralen Gruppe von Gebäuden bestand, die aus Lehmziegeln auf sorgfältig aus Quadern konstruierten Steinsockeln errichtet wurden $^{56}$. Wie im pannonischen Limesvorland wurde auch im Fezzan römische Bautechnik angewandt ${ }^{57}$. Die Übernahme ging jedoch auch in Gerama noch weiter: Bei Ausgrabungen in Nähe des Gebäudes GER 1.3 wurde seit 2001 eine größere Anzahl von Hypokaust-Ziegeln und Tubuli zusammen mit Fragmenten von bemaltem Wandputz und hydraulischem Zement gefunden ${ }^{58}$. Die Funde belegen somit zweifelsfrei, dass es mitten in Gerama eine Thermenanlage römischen Stils samt einer Hypokaustenheizung und Wasserbecken gegeben haben muss. Nichts weist daraufhin, dass diese Spezialformen von Ziegeln und Mörtel lokal produziert wurden, das Baumaterial muss also zumindest zum überwiegenden Teil von der Africa proconsularis durch die Wüste in den Fezzan transportiert worden sein.

Zur gleichen Zeit wurden im Fezzan weitere neue Techniken eingeführt, die sich jedoch auf einem vollständig anderen Niveau abspielten. So wurde die Reibmühle, die lokal seit dem Neolithikum im Gebrauch war, im Fezzan seit dem späten 1. Jahrhundert n. Chr. durch die Drehmühle ersetzt oder zumindest ergänzt ${ }^{59}$. Da weder in der Sahara noch in Afrika südlich der Sahara diese

Reynolds - John Dore, Synthesis of Human Activities in Fazzan, in: Mattingly, Synthesis, S. 327-373; David J. Mattingly - Andrew I. Wilson, "Concluding Thoughts: made in Fazzan?" in The archaeology of Fazzan. Volume 3, Excavations of C.M. Daniels, hrsg. David J. Mattingly (London - Tripoli, 2010) S. 523-530 (im Folgenden zitiert als Mattingly, Excavations).

56 Zu Gerama: Mahmoud S. Ayoub, Excavations at Germa, the Capital of the Garamantes. Preliminary Report (Tripoli, 1962); ders., Excavations in Germa between 1962 and 1966 (Tripoli, 1967); ders., The Rise of Germa: 100-450 A.D., in Libya in History. Historical Conference 16.-23. March 1968, hrsg. Fawzi F. Gadallah (Benghazi, 1968) S. 194-204; Ruprechtsberger, Garamanten, S. 30-36; David J. Mattingly u. a., in The Archaeology of Fazzan. Volume 2, Site Gazetteer, Pottery and Other Survey Finds, hrsg. David J. Mattingly (London - Tripoli, 2007) S. 115-118 .

57 David Mattingly, "Fortifications, settlement and domestic architecture," in Mattingly, Synthesis, S. 163-165; Andrew Wilson, "Saharan Trade in the Roman Period: Short-, Medium- and Long-Distance Trade Networks," Azania: Archaeological Research in Africa 47 (2012), 427.

$5^{8}$ David Mattingly, "Fortifications, Settlement and Domestic Architecture," in Mattingly, Synthesis, S. 165; Wilson, "Trade," S. 428.

59 Mattingly, Synthesis, S. 36o; David J. Mattingly, Andrew I. Wilson, "Concluding Thoughts: Made in Fazzan?," in Mattingly, Excavations, S. 528; Wilson, “Trade," S. 428. 
Art von Mühle so früh bezeugt ist, muss diese technische Weiterentwicklung aus dem Imperium Romanum stammen.

Eine vergleichbare technische Aneignung fand auch im Bereich der Textilverarbeitung statt. In jüngster Zeit wurden in Saniat Jibril und Aghram Nadarif Webgewichte entdeckt, wodurch der Gebrauch des Gewichtswebstuhls seit dem 1. Jahrhundert $n$. Chr. für den Fezzan gesichert ist ${ }^{60}$. Auch hier ist die Einfuhr durch den Austausch mit dem Imperium Romanum am wahrscheinlichsten. Weitere Funde zeigen, dass der Gewichtswebstuhl bis in das 4. Jahrhundert im Fezzan üblich war ${ }^{61}$. Interessant ist dabei, dass die nächste Stufe in der Entwicklung des Webstuhls, der Rahmenwebstuhl, nicht eingeführt wurde, obwohl dieses Gerät, bei dem ein zweiter Webbalken die Webgewichte ersetzte, im Imperium Romanum bereits seit dem späten 1. Jahrhundert $\mathrm{n}$. Chr. den Gewichtswebstuhl verdrängte ${ }^{62}$. Anscheinend war der technologische Fortschritt und die dadurch bedingte komfortablere Handhabung für die Garamanten nicht von entscheidender Bedeutung, oder die Neuerung gelangte aus unbekanntem Grund nicht mehr in den Fezzan ${ }^{63}$.

\section{$2.4 \quad$ Reich Herodes des Großen}

Weitere Beispiele fürTechnologietransferstammen aus dem Herrschaftsbereich Herodes des Großen, also aus einem der während der späten Republik und der frühen Kaiserzeit recht häufigen Klientelstaaten in der östlichen Peripherie des Imperium Romanum ${ }^{64}$. Im Zentrum des Interesses steht der zweite

$60 \quad$ Saniat Jibril: David J. Mattingly - John Hawthorne - Charles M. Daniels, in: Mattingly, Excavations, S. 194-197 Abb. 3.83; 3.84; Aghram Nadharif: L. Mori, "The Loom Weights," in Aghram Nadharif. The Barkat Oasis (Sha'abiya of Ghat, Libyan Sahara) in Garamantian times, hrsg. Marco Liverani (Florenz, 2005) S. 319-321; Wilson, "Trade," S. 428.

61 David J. Mattingly, Andrew I. Wilson, “Concluding Thoughts: Made in Fazzan?," in Mattingly, Excavations, S. $5^{28}$.

62 Zur Technologie von Webstühlen im Imperium Romanum zusammenfassend: John P. Wild, "Textile Production," in The Oxford Handbook of Engineering and Technology in the Classical World, hrsg. John P. Oleson (Oxford, 2008) S. 465-482 (mit Belegen).

63 Weites wurden Weinpressen mediterranen Typs im Fezzan eingeführt: Wilson, "Trade," S. 428.

64 Nikos Kokkinos (Hrsg.), International Conference The World of the Herods and the Nabataeans: held at the British Museum, 17-19 April 2001. 1. The World of the Herods (Stuttgart, 2007); ,Zur materiellen Kultur im Klientelreich Herodes' des Großen: Günther Schörner, „Rom jenseits der Grenze: Klientelkönigreiche und der Impact of Empire,“ in Frontiers in the Roman World. Proceedings of the Ninth Workshop of the International Network Impact of Empire, hrsgg. Olivier Hekster, Ted Kaizer (Hrsg.), (Leiden - Boston 2011) S. 124-130. 
Winterpalast Herodes des Großen bei Jericho im Jordantal, der zusammen mit den hasmonäischen und zwei weiteren herodianischen Bauten von 1973 bis 1987 aufgedeckt wurde ${ }^{65}$. Die Anlage wurde nach einem Erdbeben im Jahre 31 v. Chr. erbaut und besteht aus zwei Teilen, die miteinander durch eine breite Treppe verbunden sind ${ }^{66}$. Das Zentrum des Nordflügels bildet ein Peristyl, während im Süden zwei Wasserbecken, ein Garten und ein Bad liegen. Dieser Trakt umfasst neben einem Korridor ein Praefurnium, ein Caldarium, ein Tepidarium, ein Frigidarium sowie eine Latrine und ist somit deutlich römischen Vorbildern verpflichtet ${ }^{67}$. Dennoch gibt es einige signifikante Abweichungen: So wurde das Warmbad zwar mit einer Hypokaustenheizung ausgestattet, doch waren die Stützpfeiler nicht wie in den meisten anderen römischen Bädern aus Ziegeln aufgemauert, sondern bestanden aus lokalem Kalkstein, so dass in diesem Punkt eine Anpassung an die lokale Baupraxis vorliegt ${ }^{68}$. Wesentlich tiefgreifender ist die folgende Adaption: Raum $\mathrm{A}(\mathrm{L})$ 346 sieht aufgrund des umlaufenden Kanals aus wie eine normale römische Latrine, doch fehlt sowohl eine Frischwasserzuführung als auch eine Abwasserableitung69. Trotz dieses gravierenden Unterschieds zu den meisten römischen Anlagen ist die Interpretation als Toilette für den Ausgräber Ehud Netzer nicht in Zweifel zu ziehen, da der Grundriss, die Lokalisierung und das Fehlen eines Raums als Alternativlösung für diese Nutzung sprechen ${ }^{70}$. Zur Erklärung der fehlenden Wasserleitungen und somit allgemein des Verzichts auf Wasser beim Betrieb der Latrine führt Netzer an, dass nach jüdischer Tradition Exkremente auf den Feldern entsorgt werden sollen, was im herodianischen Palast mit Hilfe von Körben oder anderen tragbaren Gefäßen geschah $^{71}$. Der Verzicht auf Wasser ist eine Folge kultureller Vorgaben, da

65 Ehud Netzer, Hasmonean and Herodian Palaces at Jericho. Final Reports of the 1973-1987 Excavations. Volume I: Stratigraphy and Architecture (Jerusalem, 2001) (im Folgenden zitiert als Netzer, Stratigraphy); jüngste Zusammenfassung zu den Palastanlagen: Kathryn Gleason, Rachel Bar-Nathan, „Paradeisoi of the Palace Complex at Jericho“, in Hasmonean and Herodian Palaces at Jericho. Final Reports of the 1973-1987 Excavations. Volume V: The Finds from Jericho and Cypros (Jerusalem, 2013) S. 325-347.

66 Netzer, Stratigraphy, S. 7f. (Datierung).

67 Zum Bad: Netzer, Stratigraphy, S. 186f. Plan 30; 211-214. Korridor: A(L) 341; Frigidarium: A(L) 345; Latrine: A(L) 346; Caldarium: E 4/1; tepidarium: E 4/2; Praefurnium: E 4/10.

68 Netzer, Stratigraphy, S. 213 Taf. 8.

69 Netzer, Stratigraphy, S. 211-212 Abb. 303. 304.

$70 \quad$ Netzer, Stratigraphy, S. 212.

71 Netzer, Stratigraphy, S. 212. Vgl. auch Zena Kamash, „Perceptions of Latrines: Sociocultural, Moral and Religious Issues," in Roman Toilets. Their Architecture and Cultural History, hrsgg. Gemma C.M. Jansen - Ann O. Koloski-Ostrow - Eric M. Moormann, 
religiös begründete Gebote und Verbote die entscheidende Begründung liefern, und keine Frage der technischen Fähigkeiten. Innerhalb eines größeren technischen Komplexes - einem Bad nach römischem Muster - wurde für eine Komponente also eine eigene kulturspezifische technische Lösung gewählt ${ }^{72}$.

\section{$3 \quad$ Übergreifende Überlegungen}

\subsection{Räumlich und zeitliche Charakteristika}

Die Ausfuhr von Waren und Praktiken, und somit auch von Techniken aus dem Imperium Romanum lässt bestimmte Regelmäßigkeiten erkennen. So zeigt die Präsenz römischer Artefakte bei Gesellschaften außerhalb des Römischen Reichs, dass diese Regionen zum Hinterland oder zumindest zur Einflusssphäre des Imperium Romanum gehörten. Für die Region nördlich der Donau im heutigen Mähren und der Slowakei prägten Jan Bouzek und Iva Ondřejová den Terminus třety zóna (dritte Zone), um die enge Verbundenheit mit dem Römischen Reich auch terminologisch zum Ausdruck zu bringen ${ }^{73}$. Folglich wurde Technologie exportiert zu oder importiert von den Gruppen, die die engsten Beziehungen und somit direkten Kontakt zu Rom hatten. Hierin besteht ein wichtiger Unterschied zur Ausfuhr römischer Objekte in das Barbaricum, der auch über Mittelsmänner erfolgen konnte. Grundsätzlich gilt jedoch, dass Technologietransfer immer auch mit einem Export von Waren verbunden ist. Geographische Nähe scheint für diese Form des Austauschs

Supplement Bulletin Antieke Beschaving 19 (Leuven, 2011) S. 186f. (im Folgenden zitiert als Jansen u. a., Toilets); dies., "An Exploration of the Relationship between Shifting Power, Changing Behaviour and New Water Technologies in the Roman Near East," Water History 4 (2012), 88. Sammlung der Quellen: Stefanie Hoss, "Jewish and Christian Texts on Ancient Latrines," in Jansen u. a., Toilets, S. 47-50; zur Situation in Palaestina allgemein: dies., "Palestine," in Jansen u. a., Toilet, S. 184f. Auch andernorts konnte vor allem im privaten Bereich auf Spülung durch Anschluss an eine Wasserleitung verzichtet werden, doch benutzte man dann Wasser, das in Eimer bereit gestellt wurde: Gemma C.M. Jansen, Antonella Merletto, „Water Use and flushing Activities,“ in Jansen u. a., Toilets, S. 73-76. (frdl. Hinweis von Richard J. Talbert).

72 Grundlegend zur Ablehnung von Technik aus kulturellen Gründen vgl. die Fallstudie: Georges Guille-Escuret, "Technical Innovation and Cultural Resistance: The Social Weight of Plowing in the Vineyards of Les Corbières (Languedoc)," in Technological Choices: Transformation in Material Cultures since the Neolithic, hrsg. Pierre Lemonnier (London New York, 1993) S. 214-226.

73 Jan Bouzek, Iva Ondřejová, „Třetí zóna“ mezi Římem a barbarikem,” Archeologické rozhledy $42 / 1$ (1990), 22-35. 
keine Voraussetzung gewesen zu sein, wie man im Fall des Fezzan sieht, der eine Karawanenreise von mehreren Wochen vom Imperium Romanum entfernt war ${ }^{74}$.

Die betrachteten Fallbeispiele für Technologietransfer fanden zu unterschiedlicher Zeit statt, im späten 1. Jahrhundert v. Chr. in Iudaea, im 1. Jahrhundert beim Bleibergbau in Germanien, im späten 1. Jahrhundert $n$. Chr. im Fezzan, in der 2. Hälfte des 3. Jahrhunderts bei der Keramikherstellung in Germanien, im 3. und den ersten Jahrzehnten des 4. Jahrhunderts für römische Bau- und Heiztechnik in der Slowakei. Der Zeitraum, in dem der Technologietransfer stattfindet, wird bestimmt durch die Intensität der Kontakte zwischen Zielregionen und Imperium Romanum: So sind die Bande zwischen Herodes und Rom evident ${ }^{75}$. Für den Fezzan sind Konflikte mit dem Imperium für das spätere 1. Jahrhundert überliefert: Die Garamanten hatten Lepcis Magna geplündert und wurden durch Gaius Valerius Festus in der Regierungszeit Vespasians, wie Tacitus schreibt ${ }^{76}$, zurückgeschlagen. Es ist deshalb plausibel, die Intensivierung der Beziehungen zu dieser Region als die Bemühungen zu interpretieren, eine Art der Vorfeldverteidigung zu etablieren ${ }^{77}$. Zweifelsfrei ist, dass in den Gräbern im Fezzan besonders viele Terra sigillata-Gefäße gefunden wurden, die in dieser Phase produziert wurden $^{78}$. Auch in Germanien folgt Technologietransfer einem ähnlichen Muster: Die Entwicklung germanischen Bleibergbaus folgt auf eine intensive

74 René Rebuffat, "Les Romains et les routes caravanières," in Le Sahara, lien entre les peubles et les cultures. Actes du Colloque organisé à Douz du 19 au 22 décembre 2002 (Tunis, 2004) S. 221-260. Vgl. hier auch den Beitrag von Wim Broekaert und Wouter Vanacker.

75 Vgl. hier Anm. 64. Hierzu gehört auch - als ein weiteres Beispiel für Technologietransfer die Verwendung von opus caementicium beim Bau des Hafens von Caesarea: Graham Branton, John P. Oleson, „The Technology of King Herod's Harbour," in Caesarea Papers. Straton's Tower; Herod's Harbour and Roman and Byzantine Caesarea, hrsg. Robert L. Vann, Suppl. Journal of Roman Archaeology 5 (Portsmouth, Rhode Island, 1992) S. 46-67.

76 Tac. Hist. IV 49.

$77 \mathrm{Zu}$ den Beziehungen zwischen Garamanten und Imperium Romanum: Mark Milburn, "Romans and Garamantes - an Enquiry into Contacts," in Town and Country in Roman Tripolitania. Papers in Honour of Olwen Hackett, hrsgg. David J. Buck - David J. Mattingly (Oxford, 1985) S. 241-261.

78 Sergio Fontana, "I manufatti romani nei corredi funerari del Fezzan. Testimonianze dei commerci e della cultura dei Garamanti (I-III sec. d. C.)," in Productions et exportations africaines: actualités archéologiques en Afrique du Nord antique et mediévale. VI Colloque Internationale sur l'Histoire et l'Archéologie de l'Afrique du Nord (Pau, octobre $1993-118^{e}$ congrès), hrsg. Pol Trosset (Paris, 1995) S. 405-420; Erwin W. Ruprechtsberger, Die Garamanten. Geschichte und Kultur eines libyschen Volkes in der Sahara. Sonderhefte Antike Welt (Mainz, 1997) S. 27.65. 
Interaktionsphase, die nicht mit der Varus-Niederlage endete, sondern unter Tiberius und seinen Nachfolgern fortgesetzt wurde ${ }^{79}$. Noch intensiver waren Kontakte zwischen Imperium und ,Germanen' in der 2. Hälfte des 3. Jahrhunderts, da eine deutliche Zunahme von Importfunden in Germanien während der Existenz des Imperium Galliarum konstatiert werden konnte ${ }^{80}$. Auch die Bauten mit römischer Steinmetz- und Heiztechnik im pannonischen Limesvorland fanden zu einer Zeit statt, als es zu Auseinandersetzungen zwischen Römern und Gruppen außerhalb des Reiches kam ${ }^{81}$. Der an sich naheliegende Schluss ist also, dass Technologietransfer in Phasen besonders intensiven Kontakts stattgefunden hat, wobei dieser durchaus militärisch gewesen sein kann.

\subsection{Modalitäten des Technologietransfers}

3.2.1 Appropriate Technology - High Technology

Neben den räumlichen und zeitlichen Gegebenheiten ist Technologietransfer durch die Modalitäten des Austausches näher zu bezeichnen ${ }^{82}$. Nicht alle Möglichkeiten, wie römische Objekte über die Grenze gelangen konnten, ist für einen solchen Austausch geeignet. So kann beispielsweise ausgeschlossen

79 Zusammenfassend: Jan Bemmann, „Das rechtsrheinische Vorland im 1. Jahrhundert n. Chr.," in Krieg und Frieden. Kelten - Römer - Germanen. Ausstellungskatalog Rheinisches Landesmuseum Bonn (Bonn, 2007) S. 97-105 (mit Belegen); Peter S. Wells, Die Barbaren sprechen. Kelten, Germanen und das römische Europa (Darmstadt 2007) S. 133-160.

80 Jan Bemman, „Romanisierte Barbaren oder erfolgreiche Plünderer? Anmerkungen zur Intensität, Form und Dauer des provinzialrömischen Einflusses auf Mitteldeutschland während der jüngeren Römischen Kaiserzeit und der Völkerwanderungszeit," in Antyk i Barbarzyncy. Ksiega dedykowana Profesorowi Jerzemu Kolendo w siedemdziesiata rocznice urodzin, hrsgg.Aleksander Bursche, Renata Ciołek (Warschau 2003) S. 53-108; Andreas Rau, „Das nördliche Barbaricum zur Zeit der Krise des 3. Jahrhunderts n. Chr. - Einige kritische Anmerkungen zur Diskussion über provinzialrömischnordeuropäische Verbindungen," in Die Krise des 3.Jahrhunderts n. Chr. und das Gallische Sonderreich. Akten des Interdisziplinären Kolloquiums Xanten 26. bis 28. Februar 20og, hrsg. Thomas Fischer, Zakmira 8 (Mainz, 2012) S. 343-430; Fallbeispiel: Christoph G. Schmidt, "Just Recycled? New Light on the Roman Imports at the ,Central Farmstead" of Frienstedt (Central Germany)," in Wells, Rome beyond its Frontiers, S. 57-70 (mit weiterer Literatur).

81 z.B. Kristian Elschek, „Römisch-germanische villae rusticae im Limesvorfeld von Carnuntum? Ergebnisse systematischer Grabung und Prospektion," in: Roman Frontier Studies 1995 (Oxford, 1997) S. 228-232. Ein wichtiges Indiz sind die zahlreichen Importe von Keramik: Klára Kuzmová, Terra sigillata im Vorfeld des nordpannonischen Limes (Südwestslowakei) (Archaeologica Slovaca Monographiae Fontes) XVI (Nitra, 1997).

82 Thomas Grane, "Roman Imports in Scandinavia: Their Purpose and Meaning?," in Wells, Rome beyond its Frontiers, S. $3^{2-35}$. 
werden, dass die Tubuli und Hypokaustanlagen im römischem Stil als Beute in den Fezzan oder die Slowakei gelangten. Tributzahlungen, Handel oder Übergabe von diplomatischen Geschenken könnten realistischere Optionen für Technologietransfer sein, doch auch das kann - zu Recht - bezweifelt werden. In seinem Artikel ,How Technology Was Transferred in the Western Provinces' nennt Kevin Greene verschiedene Optionen als Medien des Transport innerhalb des Römischen Reichs ${ }^{83}$ : Fachliteratur wie ein Beispiel in Vitruvs ,Zehn Büchern über Architektur‘ auf uns gekommen ist, Handbücher für die Provinzialverwaltung, deren Existenz postuliert wird, auch wenn kein Beispiel erhalten blieb, private Firmen und vor allem das römische Militär. Von besonderer Bedeutung ist seine Feststellung, dass Technik nicht nur aus Geräten und Maschinen besteht, sondern auch aus Menschen, die diese bedienen können, so dass sich Technologietransfer nicht im Transport von Dingen erschöpft haben kann, sondern soziale Verbindungen vorausgesetzt werden müssen, die freilich heute für uns nur noch anhand ihrer materiellen Hinterlassenschaften erkennbar sind ${ }^{84}$.

Bei der Betrachtung von Technologietransfer wird eine Frage besonders virulent: Soll ein interner oder ein externer Standpunkt eingenommen werden? Oder: Ist von Technologie-export oder von Technologieimport zu sprechen? Eng verbunden ist die Frage nach der Handlungsmacht: Wer war verantwortlich für den Technologietransfer? Diese Fragen sind zwar für alle Formen der Übernahme und Adaption von Dingen von entscheidender Bedeutung, doch werden sie bei grenzüberschreitenden Praktiken besonders virulent, da Personen bzw. Gruppen involviert sind, die in ganz unterschiedlichen Räumen und unterschiedlichen sozialen Kontexten verortet sind.

Zur besseren Einschätzung, wie römische Technologie transportiert wurde, wie sie übernommen wurde und wie sie an lokale Bedürfnisse und lokale Vorstellungen angepasst wurde, sind moderne Forschungen zum Technologietransfer eine wichtige Quelle für Anregungen. Als geeignet erwiesen sich insbesondere Überlegungen, die im Rahmen der Entwicklungshilfe gemacht wurden, da sie zum einen von einem größeren technologischen

83 Kevin Greene, "How Technology Was Transferred in the Western Provinces," in Current Research on the Romanization of the Western Provinces, hrsgg. Mark Wood, Francisco M.V.R. Queiroga (London, 1992) S. 101-105.

$84 \mathrm{Zu}$ Netzwerken bei römischen Importen in das Barbaricum: Peter S. Wells, "Roman Imports in a Larger Context: Some Questions for Further Research," in Wells, Rome beyond its Frontiers, S. 123f. (mit Beispielen). 
Gefälle zwischen Ursprungs- und Nutzungsort ausgehen und zum anderen die Rolle der indigenen Gesellschaften betonen ${ }^{85}$.

Heuristisch besonders weiterführend ist insbesondere die Unterscheidung zwischen appropriate technology und high technology. Appropriate technology oder angemessene Technologie ist ein Begriff, der in den 7oer Jahren des 20. Jahrhunderts geprägt wurde ${ }^{86}$. Auf der Website der FAO, der Ernährungs- und Landwirtschaftsorganisation der UNO, wird diese angemessene Technologie definiert ${ }^{87}$ : „It can best be defined as fulfilling two essential criteria: 1) it must be sustainable; and 2) it must be locally accepted and adapted. Sustainability implies that whatever implement, tool or machine is added to a system to improve efficiency should be locally available, or can be produced locally. $[\ldots . . . . "$

Im Kontrast dazu ist high technology oder Hochtechnologie als besonders kostenintensiv und für die Gesellschaft, in der die Technologie transportiert wird, als besonders fremdartig zu sehen. Obwohl es sich in beiden Fällen um moderne Termini handelt, lassen sie sich gut auf unsere Fallstudien anwenden und helfen, Modi der Verbreitung und der Adaption sowie allgemein die Akzeptanz zu verstehen. Allgemein sind zwei Fragen für eine entsprechende Qualifizierung zu beantworten:

1.) Sind die vermittelten Technologien nachhaltig und wenn ja, in welchem Umfang?

2.) Wurden sie von den lokalen Gruppen angenommen und übernommen?

Die Fallstudien konnten freilich auch zeigen, dass nicht immer eindeutige Entweder-Oder-Entscheidungen getroffen werden können, sondern dass der Übergang von angemessener Technologie zur Hochtechnologie fließend sein kann, und deshalb die Einteilung zu schematisch ist. Zu fragen ist deshalb, wie

85 Ein Musterbeispiel für erfolgreichen Technologietransfer in der Moderne, der nicht nur auf technische, sondern auch auf soziale und kulturelle Aspekte eingeht, stellt die Simbabwe-Buschpumpe dar: Marianne de Laet, Annemarie Mol, „The Zimbabwe Bush Pump. Mechanics of a Fluid Technology," Social Studies of Science 30 (2000), 225-263.

86 Peter Dunn, Appropriate Technology: Technology with a Human Face (London, 1978); Greene, Technology Transferred," S. 102. Zu appropriate technology auch: Thomas P. Hughes, "The Evolution of Large Technological Systems," in The Social Construction of Technological Systems: New Directions in the Sociology and History of Technology, hrsgg. Wiebe Bijker, Thomas Hughes, Trevor Pinch (Cambridge, Massachusetts - London, 1987) S. 68-70.

87 <http://www.fao.org/docrep/x5596e/x5596eo6.htm\#TopOfPage> (o6.01.2014). 
Technologien, ihr Transfer und ihre Rezeption genauer charakterisiert werden können.

In Anlehnung an Arbeiten aus dem Feld der Science and Technology Studies, aber auch aus dem Bereich der Behavioral Archaeology, wurde deshalb ein Fragenkatalog entwickelt, um die Intensität der Einbindung und Auswirkungen der übernommenen Technologie auf die indigenen Gruppen besser einschätzen zu können ${ }^{88}$.

1.) Wurde die Technologie von lokalen Gruppen in größerem Maßstab angewandt und imitiert oder wurde sie nur von kleinen fremden Gruppen, z.B. vor Ort präsenten Römern, ausgeführt oder betrieben?

2.) Wurde die Technologie weiterverbreitet oder blieb sie ein einmaliges Phänomen?

3.) Wurde die neue Technologie dazu benutzt, lokal oder regional vorhandene Einrichtungen oder Geräte zu verändern oder zu verbessern, das heißt wurde sie in lokale Praktiken integriert oder war sie ein isolierter Fremdkörper?

4.) Können wir eine Verschiebung oder Modifikation in der Anwendung der Technologie feststellen? Können wir auf eine aktive und produktive Beschäftigung von Indigenen mit der Technologie schließen oder wurde sie nur passiv rezipiert?

Als besonderer Fall: Können wir eine Verschiebung oder Modifikation in der Organisation der Technologie feststellen?

5.) Wurden lokal oder regional verfügbare Produkte oder Rohstoffe für die Anwendung verwendet oder wurde die Technologie als fremdes Fertigprodukt implantiert?

6.) Wurde die neue Technologie lange genutzt oder war sie ein kurzfristiges Phänomen?

Die Beantwortung dieser Fragen im Rahmen unserer Fallstudien hilft, die appropriateness, die Angemessenheit der übernommenen Technologien für die empfangenden Gesellschaften zu evaluieren, auch wenn natürlich nicht immer eine definitive Antwort auf die Fragen möglich ist.

88 z. B. Michael Brian Schiffer, "Studying Technological Change: a Behavioral Perspective," World Archaeology 36 (2004), 579-585, vor allem 582; Wiebe E. Bijker, "How is Technology Made? - That is the Question!," Cambridge Journal of Economics 34 (2010), 63-76, besonders 72 . 
Die Übersichtstabelle (Abb. 7.1) macht die Bandbreite deutlich, wie die Antworten auf die Fragen zu kombinieren sind, und zeigt, dass Technologietransfer ein vielgestaltiger Prozess ist. Als sehr gute Beispiele für appropriate technology, für angemessene Technologie, können der Gewichtswebstuhl und die Drehmühle im Fezzan gesehen werden, da sie lange im Gebrauch waren, für sie lokale Rohstoffe genutzt wurden, sie innerhalb des Fezzan häufig auftreten und sie nachhaltig das Weben und Mahlen in der gesamten Region veränderten. Auf der anderen Seite der Skala liegen Technologien, die am besten als Hochtechnologie bezeichnet werden können. Beispiele hierfür sind die Нypokaustheizungen und Badeanlagen im pannonischen Limesvorland und im Fezzan, die nicht adaptiert werden und ein isoliertes Phänomen bleiben. Die Technik ist im Falle des Bades von Bratislava-Dúbrovka so fremd, dass sie nur ganz oberflächlich imitiert wird und eine Badeattrappe entsteht. Ein ähnlich isoliertes Phänomen ist die Töpferwerkstatt in Haarhausen, für die vor allem die sehr kurze Lebensdauer bezeichnend ist. Deutlich ist jedoch zwischen den mit der Töpferei verbundenen verschiedenen Einzeltechniken - Bau von Töpferöfen, Verwendung der Drehscheibe und Brennen der Gefäße - zu unterscheiden, da nach Aufgabe des Produktionsortes Haarhausen zumindest die Töpferscheibe im Gebrauch bleibt. Wichtig ist auch, dass vor Ort vorhandenes Material, nämlich Ton, verwendet wurde und die Brenntechnik insofern den lokalen Gegebenheiten angepasst wurde, als anstelle einer oxydierenden eine reduzierende Atmosphäre beim Brand hergestellt wurde, so dass die Färbung der Endprodukte sich deutlich von römischen Erzeugnissen unterschied. Zumindest partiell wurde somit in Teilbereichen die an sich römische Technologie adaptiert. Die Befunde in Haarhausen sind durch die neueren Untersuchungen zur Töpferscheibenkeramik in Mainfranken zu ergänzen, soweit es Gefäßherstellung betrifft. Von besonderer Bedeutung sind die modifizierten Reibschalen, da sie Hinweise liefern, dass nicht nur entweder in römischer oder in germanischer Tradition produziert wurde - und dies ohne gegenseitige Beeinflussung -, sondern dass auch die so typisch römischen mortaria Veränderungen unterworfen werden, also eine lokale Adaption vorgenommen wird ${ }^{89}$. Dass auch relativ komplizierte technische Vorgänge nicht immer als

89 Dies ist natürlich - wie insgesamt die Verwendung von mortaria - kein Beleg für eine ,Romanisierung', so jedoch Dietwulf Baatz, „Reibschale und Romanisierung," Acta Rei Cretariae Romanae Fautores 17/18 (1977), 147-148; Sigrid Dušek, „Römische Reibschalen im germanischen Thüringen," Alt-Thüringen 24 (1989), 183-198; Dušek, Haarhausen, S. 144-146; Morten Hegewisch, "Germanische Adaptionen römischer Importgefäße," Bericht der Römisch-Germanischen Kommission 86 (2005), 302-310. Anders zu Recht: Steidl, „Drehscheibenkeramik“, S. 87f. 110. 


\begin{tabular}{|c|l|l|l|l|l|l|}
\hline & $\begin{array}{l}\text { indigene } \\
\text { Anwendung }\end{array}$ & Weiterverbreitung & $\begin{array}{l}\text { Integration in } \\
\text { indigene Technik }\end{array}$ & $\begin{array}{l}\text { Modifkation der } \\
\text { fremden Technik }\end{array}$ & $\begin{array}{l}\text { Verwendung lokaler } \\
\text { Produkte/Rohstoffe }\end{array}$ & Dauer \\
\hline Cifér-Pác: Heizung & & & & & & \\
\hline Cifér-Pác: Häuser & & & & & & \\
\hline Dúbravka: Bad & & & & & & \\
\hline Haarhausen: Ofen & & & & & & \\
\hline Haarhausen: Brand & & & & & & \\
\hline Haarhausen: Gefäße & & & & & & \\
\hline EBleben: Gefäße & & & & & & \\
\hline Brilon: Blei & & & & & & \\
\hline Fezzan: Bad & & & & & & \\
\hline Fezzan: Webstuhl & & & & & & \\
\hline Fezzan: Mühle & & & & & & \\
\hline Jericho: Bad & & & & & & \\
\hline Jericho: Latrine & & & & & & \\
\hline
\end{tabular}

ABBILDUNG 7.1 Übersicht zu Modalitäten des Techniktransfers nach dem entwickelten Fragenkatalog.

high technology bezeichnet werden müssen, ist am besten an der germanischen Bleiverhüttung im Sauerland zu erkennen.

\subsubsection{Conceptual Transfer - Complete Transfer}

Eine weitere Kategorisierung des Transferprozesses ermöglicht das Aufgreifen eines Begriffspaars, das den Transport thematisiert: Die Unterscheidung zwischen complete transfer (kompletter Transfer) und conceptual transfer (konzeptueller Transfer) wurde von Rafael Frankel für Studien zur antiken Technikgeschichte im Kontext von Weinpressen im heutigen Israel etabliert ${ }^{90}$. Kompletter Transfer bezeichnet die Implementierung von Technologien, die im Vergleich zum Herkunftsland nicht verändert wurden. Im Kontrast dazu meint konzeptueller Transfer die Übernahme einer Technik bzw. eines technischen Vorgangs ohne gleichzeitiger Festlegung auf die materielle Umsetzung, so dass Veränderungen in Form, Größe oder Material bei der Herstellung von Geräten und Anlagen, um das technische Konzept zu realisieren und zum Funktionieren zu bringen, möglich und dementsprechend häufig sind. Beispiele für konzeptuellen Transfer unter den betrachteten Fallstudien sind die Drehmühle und der Gewichtswebstuhl, da hier nur das Konzept - Mahlen

90 Rafael Frankel, „Ancient Technologies: Complete vs. Conceptual Transfer“, Tel Aviv 39 (2012), $115^{-126 .}$ 
von Getreide durch einen drehenden Stein bzw. Spannen der Kettfäden durch Gewichte - vorgegeben war, während die materielle Umsetzung, um das Konzept zum Funktionieren zu bringen, nicht vorgegeben war. Die Varianz in der Realisierung einer Technologie kann auch bei der Umsetzung komplizierterer Vorgehensweisen, z.B. beim Herstellen von Blei, relativ groß sein, während Techniken wie die Heizung durch Erwärmen der Luft unter dem Fußboden nur einen relativ geringen Spielraum lässt, der jedoch auch ausgeschöpft wird, so wie in Jericho lokal anstehender Stein und nicht Ziegel für die Stützpfeiler verwendet wurde.

\subsubsection{Practical Technology - Prestige Technology}

Ein weiterer entscheidender Punkt für den Transfer ist das technologische Gefälle. Die technologischen Rahmenbedingungen (frames) zwischen Ursprungsgesellschaft und Zielgesellschaft müssen weitgehend übereinstimmen ${ }^{91}$. Dies ist insbesondere für das Imperium Romanum und viele seiner Nachbarn von Bedeutung, da zwischen ihnen die Unterschiede im zivilisatorischen Entwicklungsstand sehr groß waren.

Sehr große Kontraste im Verständnis und in der Anwendung von Technik erschweren den Transfer und verhindern insbesondere die Übernahme von zu komplizierter oder aufwändiger Technologie. So war die Adaption der römischen Drehmühle im Fezzan nur möglich, weil der technologische Abstand zwischen diesem Typ und der Reibmühle relativ gering war. Man bewegte sich somit im selben Rahmen. Hätte man dagegen eine Anlage mit mehreren hintereinander gestaffelten Wassermühlen wie in Barbegal in der Provence in den Fezzan transferiert, so hätte diese Technologie nicht in dem Maße adaptiert werden können, weil vielleicht die natürlichen Voraussetzungen nicht vorhanden waren, aber sicher, weil die Wassermühle eine ganz andere Komplexitätsstufe repräsentiert ${ }^{92}$. Trotzdem kommt natürlich ein solcher kompletter Transfer von weit entwickelten Technologien vor, wie die Töpferwerkstatt in Haarhausen oder die Anlagen in der Slowakei beweisen. In der Moderne wird dieses Verfahren vor allem aus ökonomischen Gründen praktiziert: Hochentwickelte Fabriken mit ganz spezifischen Fertigungstechniken werden beispielsweise in Ländern der so genannten Dritten Welt errichtet,

91 Zum Begriff des technological frame: Hans K. Klein, Daniel Lee Kleinman, "The Social Construction of Technology: Structural Considerations," Science, Technology \& Human Values 27 (2002), 40.

92 Zu Barbegal: Pierre Leveau, "The Barbegal Water Mill in its Environment. Archaeology and the Economic and Social History of Antiquity," Journal of Roman Archaeology 9 (1996), 137-153. 
weil entweder lokale Rohstoffressourcen ausgebeutet werden sollen oder die niedrigeren Fertigungskosten einen entsprechenden Anreiz bieten. Dies trifft jedoch für die römischen Beispiele nicht zu: Die Töpfereien in Haarhausen oder Eßleben/Frankenwinheim wurden nicht installiert, um billiger für das Imperium zu produzieren. Ein relativ moderner Grund für die Gründung von Haarhausen wurde zwar von der Ausgräberin Sigrid Dušek genannt, nämlich das Erschließen neuer Absatzmärkte, doch sind nicht-ökonomische Aspekte entscheidender, nämlich Technologietransfer zur Repräsentation ${ }^{93}$. Diesem Aspekt kann durch eine weitere terminologische Unterscheidung Rechnung getragen werden, so dass - in Anlehnung an den Titel eines wichtigen Aufsatzes zur Technikgeschichte - von praktischen bzw. Prestige-Techniken gesprochen wird ${ }^{94}$. Grundsätzlich war der Zugang zu und die Verwendung von römischer Technik außerhalb des Imperium Romanum nicht neutral, sondern sozial bedeutungsvoll. Viele der Fallstudien sind mit lokalen Potentaten zu verbinden, wie die Latrine und das Badehaus in Jericho mit Herodes dem Großen. Auch die aufwändigen Gebäude in Cifér-Pác und Bratislava-Dúbrovka wurden wohl im Auftrag von Angehörigen der einheimischen Elite errichtet, um den eigenen sozialen Status sowohl zum Ausdruck zu bringen als auch zu erhöhen. Gleiches ist für das Bad im Kontext der aufwändigen Stadthäuser von Gerama im Fezzan anzunehmen. Ebenfalls ist für die Töpferei von Haarhausen eine soziale Funktion anzunehmen, wobei die Gefäße nicht so sehr für eigene repräsentative Zwecke benutzt wurden, sondern eher um Verbindungen innerhalb eines begrenzten Raumes materiell greifbar zu machen und auch zu verstärken. Selbst für die Bleiverarbeitung in Westfalen ist eine Kontrolle durch einen lokalen Potentaten denkbar, wie dies von Michael Erdrich vermutungsweise vorgeschlagen wurde ${ }^{95}$. Transfer römischer Technologie über die Grenzen hinaus ist also nicht nur Ausdruck der Beziehungen zwischen provinzialen und indigenen Gesellschaften, sondern kann auch innerhalb dieser Gruppen wichtige soziale Funktionen erfüllen.

93 Dušek, Haarhausen, S. 132-148.

94 Brian Hayden, "Practical and Prestige Technologies: The Evolution of Material Systems," Journal of Archaeological Method and Theory 5 (1998), 1-55. Der Status erhöhende Effekt wird konkret benannt bei einem weiteren Beispiel von Technologietransfer: Birgit Arrhenius, „Die Schraube als Statussymbol. Zum Technologietransfer zwischen Römer und Germanen," in Frankfurter Beiträge zur Mittelalterarchäologie II. Schriften des Frankfurter Museums für Vor- und Frühgeschichte 12 (Frankfurt 1990) S. 9-26.

95 Michael Erdrich, „Rezension zu Walter Melzer - Torsten Capelle (Hrsg.), Bleibergbau und Bleiverarbeitung während der römischen Kaiserzeit im rechtsrheinischen Barbaricum,“ Germania 87 (2009), 629. 


\section{$4 \quad$ Schluss}

Technologietransfer jenseits der Grenzen des Imperium Romanum war ein geläufiges und vor allem ein vielfältiges Phänomen. Gemäß der Science and Technology Studies sollten Technologien als Artefakte betrachtet werden, so dass sie in Relation zu anderen Artefakten, die aus dem Imperium Romanum in das Gebiet jenseits der Grenze gebracht wurden, gesetzt werden können. Bei diesem Vorgehen bietet der Transfer von Technologien als komplexen Artefakten Analysemöglichkeiten, die beim so genannten römischen Import in das Barbaricum nicht immer gegeben sind. So lassen sich Techniken und die damit verbundenen Gerätschaften und Strukturen viel besser hinsichtlich ihres Gebrauchs ansprechen, so dass auch genauer zu beurteilen ist, wie stark sie verändert oder angepasst wurden ${ }^{96}$. Wie bei den Fallbeispielen gezeigt können Abstufungen in der Akzeptanz, unterschiedliche Formen der Adaption und Kriterien für die Übernahme identifiziert werden, so dass dadurch viel besser gefasst werden kann, wie Kulturkontakte genauer abliefen.

96 Vgl. hierzu das Modell für den Lebenszyklus (use life) von römischen Importen: Fraser Hunter, „The Lives of Roman Objects beyond the Frontier," in Wells, Rome beyond its Frontiers, S. 15-23 mit Abb. 1. 